\title{
Sensing pH in a Microfluidic Channel with a Lab-on-a- Smartphone Fluorescence Spectrometer
}

\author{
Jiajie Chen ${ }^{1}$, Ruoyu Wang ${ }^{1}$, Abhishek Renganathan ${ }^{1}$, Anurag Rattan ${ }^{1}$, Xiangyue Meng ${ }^{1}$, Ranjith \\ Rajasekharan Unnithan ${ }^{1}$, and Kenneth B. Crozier ${ }^{1,2}$ \\ ${ }^{\prime}$ Department of Electrical and Electronic Engineering, University of Melbourne, Victoria 3010, Australia \\ ${ }^{2}$ School of Physics, University of Melbourne, Victoria 3010, Australia \\ Corresponding Author e-mail address: kenneth.crozier@unimelb.edu.au Tel: +61383442249
}

\begin{abstract}
We demonstrate a smartphone unit for measuring $\mathrm{pH}$. The dye-mixed sample is input to a microfluidic chip and inserted into the unit. The smartphone image sensor records the fluorescence spectrum, and an app determines $\mathrm{pH}$. OCIS codes: (170.2520) Fluorescence microscopy; (230.0230) Optical devices
\end{abstract}

\section{Introduction}

Due to their widespread adoption across many demographic groups and their advanced capabilities for imaging, computation and communication, there is much interest in the use of smartphones for health monitoring applications $[1,2]$. Fluorescence sensing has proven particularly useful for biology and medicine. Traditionally, however, fluorescence spectra are measured with spectrometers that are bulky, expensive, and appropriate for operation only by trained professionals. This makes them unsuitable for health monitoring in resource-limited settings such the home or in small clinics. The ability to extract the necessary information from small volume patient samples is also highly advantageous. Microfluidic devices have shown tremendous potential in this regard, due to their ability to precisely manipulate sub-microliter volumes of fluids in channels and reaction chambers [3]. In this paper, we demonstrate a lab-on-a-smartphone platform to measure the $\mathrm{pH}$ of saliva, which is an important indicator of dental health and plays an important role in caries prevention [4]. We produced a plastic smartphone unit for an iPhone 5s by 3D printing. The unit contains a laser diode (wavelength: $405 \mathrm{~nm}$ ) to illuminate the polydimethylsiloxane (PDMS) microfluidic chip containing a channel $(\sim 30 \mu \mathrm{l}$ volume $)$ serves as the sample container. Measurement proceeds as follows. The sample is mixed with the dye 5(6) carboxynaphthofluorescein (CNF), whose fluorescence spectrum varies with $\mathrm{pH}$. The mixture is input to the microfluidic chip, which is then slotted into the unit. The fluorescence emission produced in response to laser diode illumination is collected, and dispersed by a transmission grating onto the smartphone image sensor. An app converts the image to a fluorescence spectrum and determines the $\mathrm{pH}$. The latter is done from the ratio of fluorescent emission at two wavelengths. In addition to $\mathrm{pH}$, we expect many applications of our smartphone unit for healthcare monitoring via fluorescence, due to our unit being inexpensive, easy to use, small and lightweight. These attributes are of particular interest to developing countries.

\section{Design of the smartphone attachment}

Our smartphone unit is shown in Figure 1. The unit is designed using the Solidworks software package (Figure 1a) and produced by $3 \mathrm{D}$ printing (Figure $1 \mathrm{~b}$ ). The unit's main body contains a violet laser diode module, a transmission grating (1200 grooves $/ \mathrm{mm}$ ), and supports the smartphone in an ergonomic manner. For convenience and flexibility, part of the optical system (two lenses \& pinhole) is housed within the "adjustable optic component" that is slotted into the main body. As shown in Figure 2a, the laser beam path is orthogonal to the fluorescence emission collection path, to prevent it from being collected with the fluorescence emission via direct reflection from the PDMS chip.

(a)

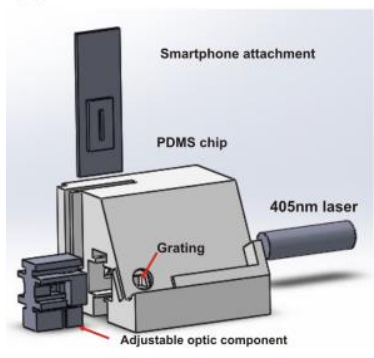

(b)

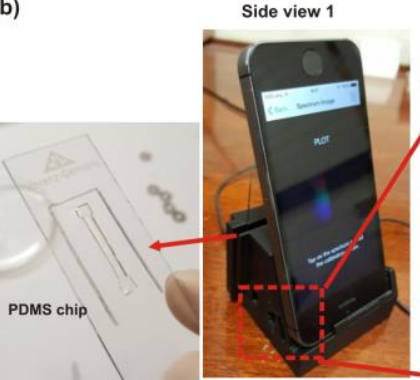

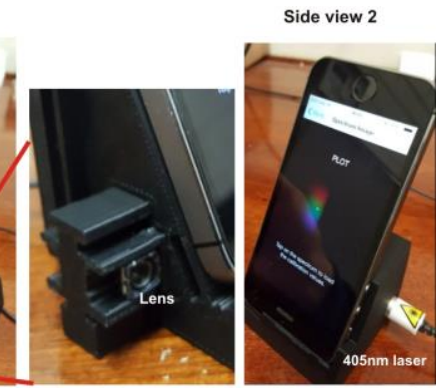

Figure 1 (a) Solidworks model of smartphone unit for fluorescence spectrum measurement. b). Photos of microfluidic chip and smartphone unit.

The PDMS mold is made by dispensing UV glue (Norland Optical Adhesive 63, volume $~ 30 \mu \mathrm{l}$ ) in a petri dish, and exposing it to UV light for 24 hours to harden it. We then mix PDMS pre-polymer with curing agent (10:1 by 
volume, Sylgard 184, Dow Corning, Midland, MI). After degassing for 3 hours, the mixture is poured onto the mold and cured at $50{ }^{\circ} \mathrm{C}$ for 12 hours. The cured PDMS replica is peeled from the master. We then treat the PDMS replica and glass side surface in oxygen plasma for 10 minutes so that they stick together tightly, thereby forming the PDMS chip. We then inject a mixture $(30 \mu \mathrm{l})$ of CNF dye $(1 \mathrm{mM})$ with the sample under study (pH buffer solution) into the channel by syringe and conduct the measurement. The mixture has CNF: sample in a volume ratio of 1:10.

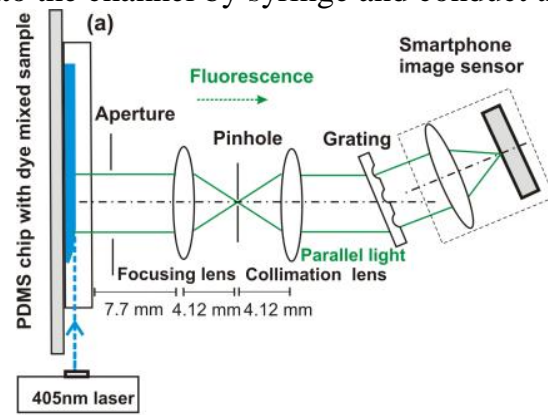

(b)

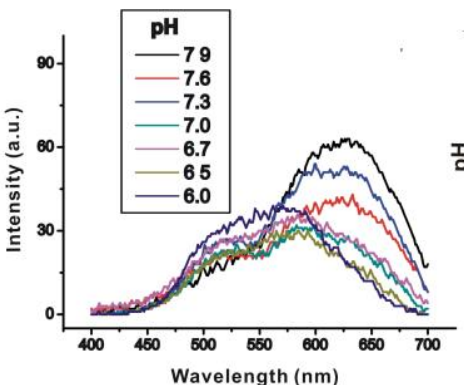

(c)

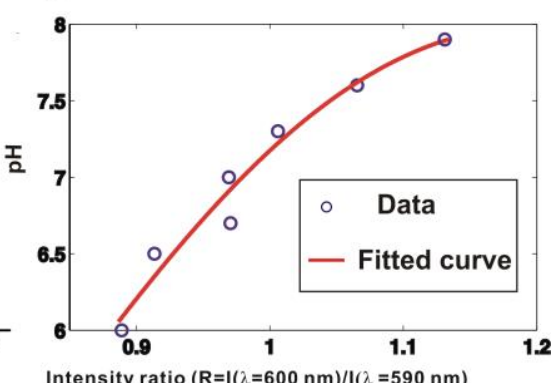

Figure 2 (a) Optical path of the smartphone unit. (b) Spectra measured for different pH values. (c) Known pH vs measured intensity ratio (R) of buffer solutions. Open circles: experimental data. Red line: fitted curve, with $\mathrm{pH}=\left(1393 \mathrm{R}^{2}-38.01 \mathrm{R}-575.2\right) /\left(\mathrm{R}^{3}+174.8 \mathrm{R}^{2}-83.2 \mathrm{R}+16.14\right)$.

\section{3. pH measurement}

Measurements of $\mathrm{pH}$ proceed as follows. Our smartphone app reads the image sensor, on which the fluorescence emission has been dispersed by the smartphone unit's optical system. Wavelength calibration of the unit is performed prior to the fluorescence measurement, using white light from an LED filtered with bandpass filters (390 $\mathrm{nm}$ and $630 \mathrm{~nm}$ ). This allows the app to then convert the image sensor image to a fluorescence spectrum (intensity vs wavelength). The intensities plotted in these spectra represent the sum of the readings of the pixels in the direction perpendicular to the spectral dispersion direction. Fluorescence spectra are measured from a number of dye-mixed pH standards (Figure 2b, Hach Company, Loveland, CO). In Figure 2c, we plot the known $\mathrm{pH}$ of the $\mathrm{pH}$ standard against the measured intensity ratio. A fitted curve is also plotted. The fit equation is used by the app to determine the $\mathrm{pH}$ of each measured sample. In Figure 3a-b, we show screen shots from the app when samples with known $\mathrm{pH}$ values of 6.5 and 7.9 are measured. A reproducibility test is presented as Figure $3 \mathrm{c}$, in which measurements are made every 2 minutes over the course of 24 minutes. Each data point takes 1-2 minutes to obtain.
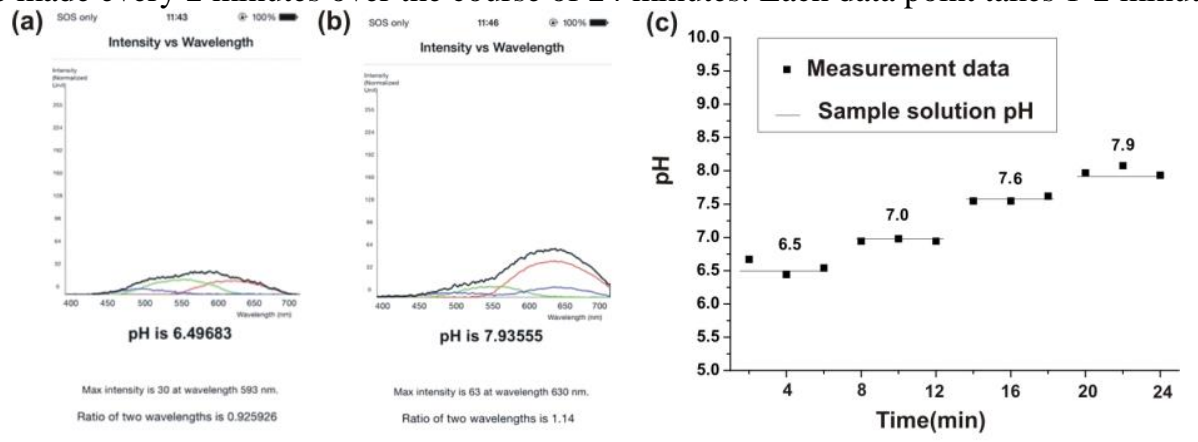

Figure 3 Smartphone measurement results of standard $\mathrm{pH}$ buffer solution with $\mathrm{pH}$ values of (a) 6.5, (b) 7.9. (c) Reproducibility test, in which data points are obtained every 2 minutes. Note that $\mathrm{pH}$ value of human saliva ranges from 6 to 8 .

Funding from Melbourne Networked Society Institute, Laby Foundation, VESKI, and Australian Research Council.

\section{Conclusion}

The smartphone unit we report has shown good reproducibility although the spectral resolution is limited by the pinhole size. We plan to improve this device by immobilizing the dye on the chip before measurement and by adding channels (and other dyes) for sensing various chemicals important for health monitoring.

\section{References}

[1] H. Zhu, I. Sencan, J. Wong, et al, “Cost-effective and rapid blood analysis on a cell-phone,” Lab on a Chip, 13, 1282-1288 (2013).

[2] V. Oncescu, D. O'Dell, and D. Erickson, "Smartphone based health accessory for colorimetric detection of biomarkers in sweat and saliva," Lab on a Chip, 13, 3232-3238 (2013).

[3] J. Wu, X. Wu, and F. Lin, "Recent developments in microfluidics-based chemotaxis studies," Lab on a Chip, 13, 2484-2499 (2013).

[4] B. P. Preethi, R. Dodawad, and A. Pyati, "Evaluation of flow rate, pH, buffering capacity, calcium, total proteins and total antioxidant capacity levels of saliva in caries free and caries active children: an in vivo study," Indian Journal of Clinical Biochemistry 25, 425-428 (2010). 